\title{
STUDIES ON THE COMPETITION OF POLYENE ANTIBIOTICS FOR STEROLS
}

\author{
J. Patterson, J. Holland and L. L. Bieber \\ Department of Biochemistry, Michigan State University \\ East Lansing, Michigan 48824, U.S.A.
}

(Received for publication January 8, 1979)

\begin{abstract}
The fractional change in the corrected fluorescence of pimaricin or filipin in the presence of a limiting amount of sterol and a competing polyene antibiotic has been used to estimate the relative affinity of amphotericin B, nystatin, filipin, and pimaricin for stigmasterol and for cholesterol. The relative affinities for cholesterol were filipin $>$ amphotericin $\mathbf{B}>$ pimaricin $>$ nystatin, while the relative affinities for stigmasterol were filipin $>$ pimaricin $>$ amphotericin $\mathrm{B}>$ nystatin. The data indicate that pimaricin and filipin can both interact simultaneously with about $30 \%$ of the cholesterol or stigmasterol. However, the stoichiometry of filipin and pimaricin alone for cholesterol and for stigmasterol in dilute aqueous solutions is $1: 1$. In the experiments which indicated both pimaricin and filipin interact with the same sterol molecule changes in corrected fluorescence and the absorbance spectra were monitored; and these criteria indicated that both pimaricin and filipin had interacted with the sterols. Lightscattering measurements indicate large aggregates were not formed. Although the data shows in dilute aqueous solutions the stoichiometry of filipin and/or pimaricin for sterols is $1: 1$, in more complex solutions, other combinations or interactions are indicated especially for pimaricin.
\end{abstract}

Membrane sterols are a target for the action of polyene antibiotics ${ }^{1)}$. However, the degree of damage caused by polyene antibiotics in natural and model membranes is variable ${ }^{2-5}$. Some of this variability may be the result of different affinities and interactions of polyene antibiotics for the membrane-bound sterols. The purpose of this investigation is to measure the relative affinities of some polyene antibiotics for cholesterol and stigmasterol.

Most of the methods for measuring the interactions of polyenes with sterols are indirect and yield a diversity of experimental results $\left.{ }^{1,6} 8\right)$. Fluorescence techniques can be used to monitor the interactions of some polyene antibiotics with sterols and give reproducible experimental results when the experimental conditions are carefully controlled ${ }^{9}$. Fluorescence data in general correlates well with the effects of polyene antibiotics on biological and model membrane systems ${ }^{10 \sim 12)}$. In dilute aqueous solutions, pimaricin and filipin exhibit characteristic changes in absorbance and fluorescence properties in the presence of sterols ${ }^{7 \sim 9,13 \sim 15)}$. The magnitude of the absorbance and fluorescence changes can be predicted for these simple systems ${ }^{9}$. We have exploited the fluorescence properties of filipin and pimaricin in an effort to determine the relative affinities of polyene antibiotics for cholesterol and stigmasterol. The results of these experiments are present herein.

\section{Materials and Methods}

Special chemicals were obtained as follows: cholesterol, stigmasterol, nystatin, and amphotericin B from Sigma Chemical Company, St. Louis, Missouri. Pimaricin was the generous gift of American Cyanamid Company, Princeton, New Jersey. Streptomyces filipinensis was kindly provided by P. G. PRIDHAM, Agricultural Service, United States Department of Agriculture, Peoria, Illinois. This organism 
was cultured and the filipin isolated as described elsewhere ${ }^{15}$. Other chemicals were reagent grade.

Stock solutions of filipin and pimaricin were prepared by dissolving $1 \mathrm{mg}$ of the polyene antibiotic in $200 \mathrm{ml}$ of distilled water with vigorous stirring at room temperature for 24 hours in the dark. Stock solutions of nystatin and amphotericin B were prepared by dissolving $2 \mathrm{mg}$ of the polyene antibiotic in $283 \mathrm{ml}$ of distilled water with vigorous stirring in the dark for 24 hours. Solutions of stigmasterol and cholesterol were prepared by dissolving the sterol in isopropanol to give the concentrations needed. The sterols were added to the polyene solutions by injection with a Hamilton syringe; specific details are given in the figure legends.

Fluorescence measurements were obtained using a computer-centered spectrofluorometer. The analytical quantity absorbance corrected fluorescence $(\mathrm{CO})$ was used in these experiments. This quantity is described in detail elsewhere ${ }^{16)}$. For all experiments, filipin was monitored at an excitation wavelength of $338 \mathrm{~nm}$ and an emission wavelength of $495 \mathrm{~nm}$. Pimaricin was monitored at an excitation wavelength of $308 \mathrm{~nm}$ and an emission wavelength of $405 \mathrm{~nm}$. All experimental points presented in the figures and tables are the average of 5 separate experiments.

\section{Results}

Competition between Filipin, Nystatin, Amphotericin B, and Pimaricin for Sterols

The purpose of these experiments was to determine the relative affinity of various polyene antibiotics for different sterols. Experiments were designed so the amounts of one polyene were varied, i.e., pimaricin; and this was added to a solution which contained a fixed amount of filipin and a fixed amount of cholesterol. For example, in Fig. 1A, the samples contained $3.8 \mu \mathrm{M}$ filipin and $2.2 \mu \mathrm{M}$ cholesterol and variable quantities of pimaricin were added. The samples were allowed to stand for 2 hours and then fluorometric and absorbance measurements were made to determine the binding of cholesterol to filipin and/or pimaricin. As shown in Fig. 1A, pimaricin, the closed circles, did not affect the fluorescence properties of filipin, the open circles, in the absence of cholesterol. However, in the presence of cholesterol, the data show that both filipin and pimaricin interacted with cholesterol even when the concentration of filipin as well as pimaricin were both greater than the concentration of cholesterol. When the mole ratio of filipin to pimaricin is 1 , the total concentration of polyene antibiotic in solution was $7.6 \mu \mathrm{M}$ and the total concentration of cholesterol was $2.2 \mu \mathrm{M}-\mathrm{a}$ threefold greater concentration of polyene antibiotic. Yet the data indicate that approximately $80 \%$ of the initial pimaricin was bound to cholesterol. When pimaricin binds to sterols, the corrected fluorescence increases; and when filipin binds to sterols, the corrected fluorescence decreases approximately $60 \%$ at full interaction. Similar types of data were obtained when: (a) the experiment was repeated, but cholesterol was replaced with stigmasterol (see Fig. 1B), and (b) when $3.8 \mu \mathrm{M}$ pimaricin in the presence of $2.2 \mu \mathrm{M}$ cholesterol or $2.2 \mu \mathrm{M}$ stigmasterol was treated with varying amounts of filipin (Figs. 1C and 1D). As shown in Fig. 1C, when the filipin/pimaricin ratio is $1: 1$, pimaricin has interacted with about $75 \%$ of the total sterol and filipin has interacted with $65 \sim 70 \%$ of the total cholesterol. Thus at a 1:1 filipin to pimaricin ratio, about $30 \%$ of the cholesterol appears to be interacting with both polyene antibiotics.

Similar experiments were done in which filipin or pimaricin and the sterol were held constant and variable amounts of nystatin and amphotericin B were added to the solutions up to a 1:1 ratio with the other polyene antibiotic. These data are given in Fig. 2. Neither nystatin nor amphotericin B effectively competed with filipin for either cholesterol (Fig. 2A) or stigmasterol (Fig. 2B), indicating a much smaller affinity for these sterols. The controls show that in the absence of sterols, neither nystatin or amphotericin B had an effect on the fluorescence properties of filipin. 
Fig. 1. The effect of different amounts of pimaricin on the competition between filipin and pimaricin for cholesterol and stigmasterol.

In $\mathrm{A}$ and $\mathrm{B}$, pimaricin $(0,1.1,2.3,3.3,4.5,5.7,7.5$, and 11.4 nmoles) were added to samples containing 11.4 nmoles of filipin in a final volume of $3 \mathrm{ml}$ in distilled water. The samples were incubated for 2 hours at $37^{\circ} \mathrm{C}$ and allowed to cool to room temperature. The samples were then assayed at an excitation wavelength of $338 \mathrm{~nm}$ and an emission wavelength of $495 \mathrm{~nm}$. The samples were also measured at an excitation wavelength of $308 \mathrm{~nm}$ and an emission wavelength of $405 \mathrm{~nm}$. The experiment was repeated, but 6.5 nmoles of cholesterol were added by injection with $10 \mu \mathrm{l}$ of isopropanol.

Closed circles: measurements made at an excitation wavelength of $308 \mathrm{~nm}$ and an emission wavelength of $405 \mathrm{~nm}$ (pimaricin).

Open circles: measurements made at an excitation wavelength of $338 \mathrm{~nm}$ and an emission wavelength of $496 \mathrm{~nm}$ (filipin).

$\triangle$ in $\mathrm{A}$ and $\mathrm{B}, 6.5 \mathrm{nmoles}$ of cholesterol were added to 11.4 nmoles of pimaricin in $3 \mathrm{ml}$ of distilled water. Samples were incubated as above and measurements were made at an excitation wavelength of $308 \mathrm{~nm}$ and an emission wavelength of $405 \mathrm{~nm}$.

$\triangle$ in $\mathrm{C}$ and $\mathrm{D}, 6.5$ nmoles cholesterol were added to 11.4 nmoles of filipin and measurements made at $338 \mathrm{~nm}$ excitation and $496 \mathrm{~nm}$ emission.

In $\mathrm{B}$, the protocol was identical to that described for $\mathrm{A}$ except stigmasterol replaced cholesterol.

In $\mathrm{C}$ and $\mathrm{D}$, the protocol was identical to that used in A and $B$ except pimaricin was held constant and different amounts of filipin were added to the solutions.

In these experiments, the maximum corrected fluorescence was set at $100 \%$ and the changes measured were relative to this.

$\diamond$ filipin (11.4 nmoles) in $3 \mathrm{ml}$ distilled water measured at $338 \mathrm{~nm}$ excitation and 495 $\mathrm{nm}$ emission.
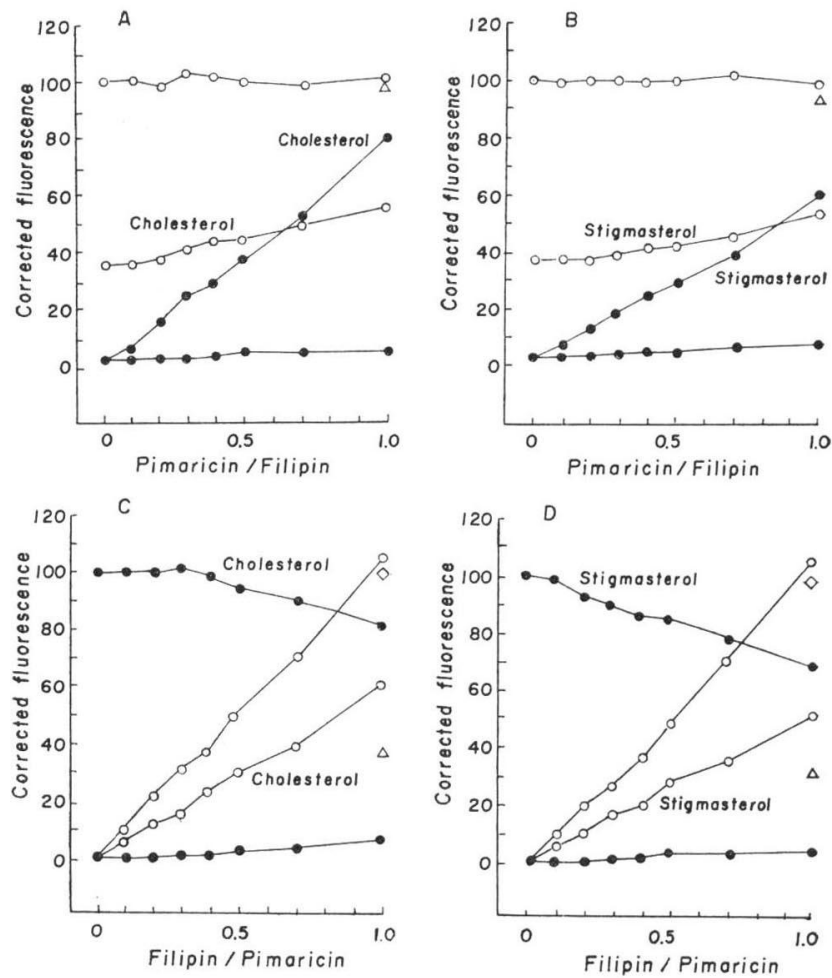

When these experiments were repeated keeping the concentration of pimaricin constant and varying the amount of nystatin or amphotericin B, different results were obtained. These data are shown in Figs. 2C and 2D. Nystatin had very little effect on the fluorescence of the pimaricin sterol complex or on the pimaricin stigmasterol complex. A small decrease in the fluorescence of the pimaricin stigmasterol complex was obtained as the nystatin concentration was increased (Fig. 2D). However, when amphotericin B was substituted for nystatin, a large decrease in the fluorescence of the pimaricin sterol complex was seen, even in the presence of very low amounts of amphotericin B (see the closed circles' top curves of Figs. 2C and 2D). Thus amphotericin B competed for either cholesterol or stigmasterol with pimaricin, however, the competition was not complete. The initial drop in pimaricin fluorescence indicates that when small amounts of amphotericin B were added, i.e., $0.4 \mu \mathrm{M}$ amphotericin B in the presence of $3.8 \mu \mathrm{M}$ pimaricin and $2.2 \mu \mathrm{M}$ cholesterol or stigmasterol, $40 \sim 50 \%$ of this sterol was removed 
Fig. 2. The effect of nystatin and amphotericin B on the fluorescence properties of filipin or pimaricin in the presence and absence of cholesterol or stigmasterol.

In $\mathrm{A}$, nystatin or amphotericin $\mathrm{B}(0,1.1,2.3,3.3,4.5,5.7,7.5$, and 11.4 nmoles $)$ was added to samples containing 11.4 nmoles of filipin in a final volume of $3 \mathrm{ml}$ of distilled water. The samples were incubated for 2 hours at $37^{\circ} \mathrm{C}$ and allowed to cool to room temperature and spectral measurements were made at an excitation wavelength of $338 \mathrm{~nm}$ and an emission wavelength of $495 \mathrm{~nm}$. The experiment was repeated with the addition of 6.5 nmoles of cholesterol which was added by injection with $10 \mu \mathrm{l}$ of isopropanol.

Open circles: filipin +nystatin, Closed circles: filipin + amphotericin B, Nys: nystatin, Amph B: amphotericin B.

In $B$ the procedure was identical to A except cholesterol was replaced by stigmasterol.

In $\mathrm{C}$ the procedure was identical to A except the filipin was replaced by pimaricin and the excitation was $308 \mathrm{~nm}$ and the emission was monitored at 405 $\mathrm{nm}$.

Open circles: pimaricin + nystatin. Closed circles: pimaricin + amphotericin B.

In $\mathrm{D}$ the procedure was identical to $\mathrm{C}$ except stigmasterol replaced cholesterol.

In $\mathrm{C}$ and $\mathrm{D}$ the maximum change in corrected fluorescence obtained by adding 6.5 nmoles of sterol to 11.4 nmoles of pimaricin was set at $100 \%$ and corrected fluorescence values were determined in the presence of amphotericin B or nystatin.
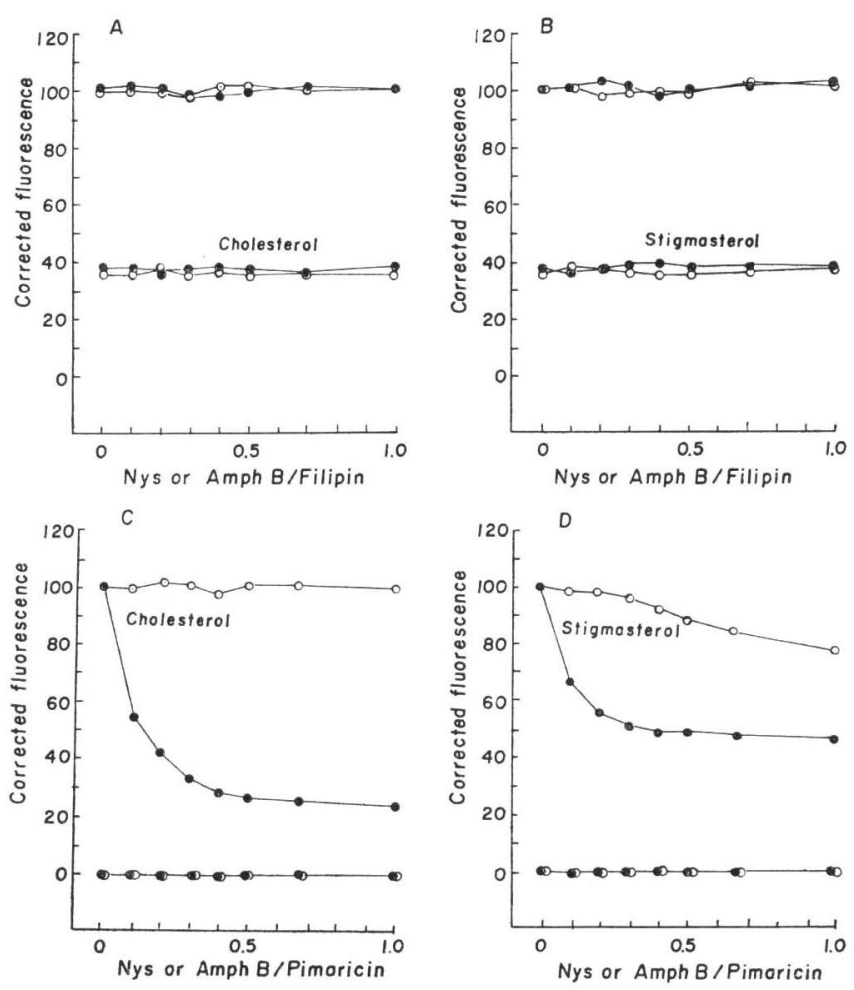

from the pimaricin sterol complex.

Sterol-Polyene Antibiotic Stoichiometry

Several interpretations of the above data are plausible. For example, one interpretation might be that with the experimental conditions used, the stoichiometry of polyene antibiotics to sterols is not 1:1. Another interpretation might be that the experimental conditions favor formation of a ternary complex, i.e., a polyene-sterol-polyene complex. Another might be the formation of a large complex with variable stoichiometries. We attempted to test some of these possibilities and consequently initiated a series of experiments to determine the stoichiometry of binding of filipin and pimaricin for stigmasterol and cholesterol using the exact experimental conditions employed for Figs. 1 and 2. These data are given in Figs. 3A $\sim 3 \mathrm{D}$ and show that the stoichiometry in dilute aqueous solutions is within experimental error $1: 1$. The molar ratio of sterol to polyene antibiotic at the break in the curve is 1.03: 1 in Fig. 3A, 1.05: 1 in Fig. 3B, 1.03: 1 in Fig. 3C, and 1.02:1 in Fig. 3D. Thus in dilute aqueous solutions with the experimental conditions used for Figs. 1 and 2, the stoichiometry of the 
Fig. 3. Titration of filipin and pimaricin with cholesterol and stigmasterol.

In $\mathrm{A}$, cholesterol $(0,1.6,3.2,5.4,6.5,8.0,13.0,21,32$, and 65 nmoles) was added by injection with $10 \mu \mathrm{l}$ of isopropanol to samples containing $11.4 \mathrm{nmoles}$ of filipin in $3 \mathrm{ml}$ of distilled water. The samples were incubated for 2 hours at $37^{\circ} \mathrm{C}$ and allowed to cool to room temperature and measured at an excitation wavelength of $338 \mathrm{~nm}$ and an emission wavelength of $495 \mathrm{~nm}$.

In $\mathrm{B}$, the procedure was identical to A except stigmasterol replaced cholesterol.

In $\mathrm{C}$, the procedure was identical to A except pimaricin (11.4 nmoles) replaced filipin and the measurements were made at $308 \mathrm{~nm}$ (excitation) and $405 \mathrm{~nm}$ (emission).

In $\mathrm{D}$, the procedure was identical to $\mathrm{C}$ except stigmasterol was substituted for cholesterol.
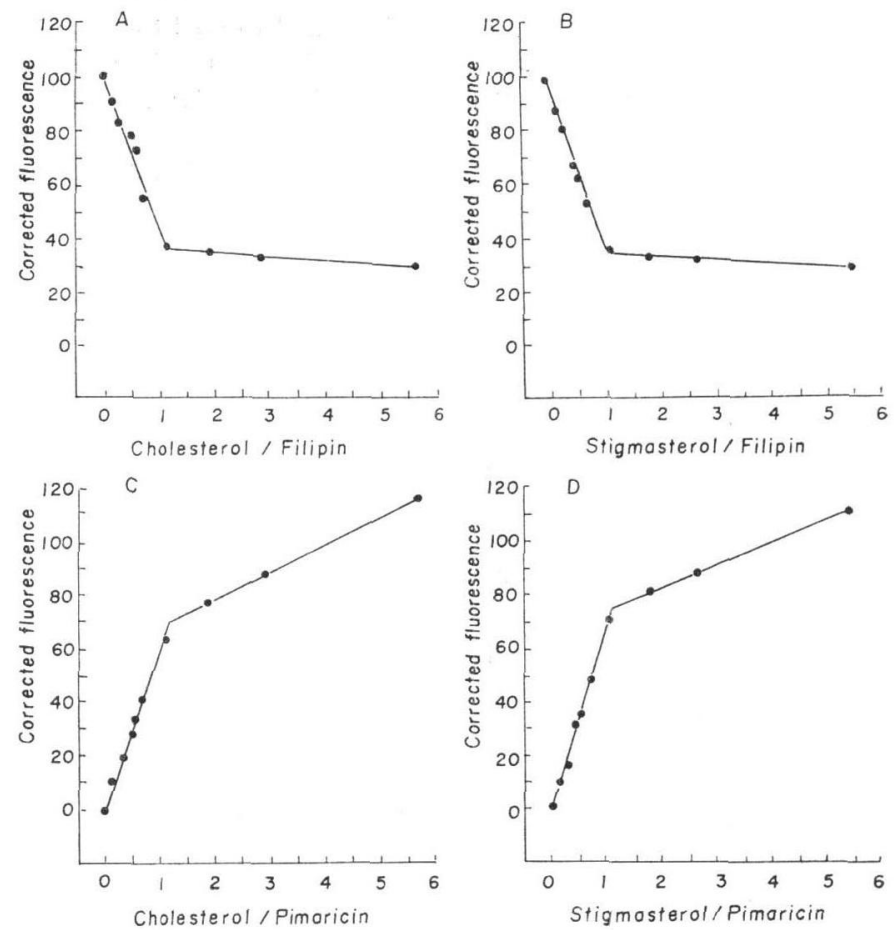

Fig. 4. Spectra of filipin and pimaricin in the presence of cholesterol and deoxycholate.

In A, filipin (11.4 nmoles) was mixed with (solid line) 11.4 nmoles of pimaricin or with (dashed line) 11.4 nmoles of pimaricin in the presence of 6.5 nmoles of cholesterol in a final volume of $3 \mathrm{ml}$ of cistilled water. The samples were incubated for 2 hours at $37^{\circ} \mathrm{C}$ and allowed to cool to room temperature before measurements were made. The excitation spectra of filipin were monitored at $495 \mathrm{~nm}$.

In B, pimaricin (11.4 nmoles) was mixed with (solid line) 11.4 nmoles of amphotericin B or with (dashed line) 11.4 nmoles of amphotericin $\mathrm{B}$ in the presence of 6.5 nmoles of cholesterol in the final volume of $3 \mathrm{ml}$ of distilled water. The samples were incubated for 2 hours at $37^{\circ} \mathrm{C}$ and allowed to cool to room temperature before absorbance spectra were taken.

In $\mathrm{C}$, pimaricin (11.4 nmoles) was mixed with (solid line) $15 \mathrm{~mm} \mathrm{Na}$ deoxycholate or with (dashed line) 11.4 nmoles of amphotericin B plus 6.5 nmoles of cholesterol. The final volume was $3 \mathrm{ml}$ in distilled water. The samples were incubated for 2 hours at $37^{\circ} \mathrm{C}$ and allowed to cool to room temperature. The excitation spectra of these mixtures were monitored at an emission of $405 \mathrm{~nm}$.
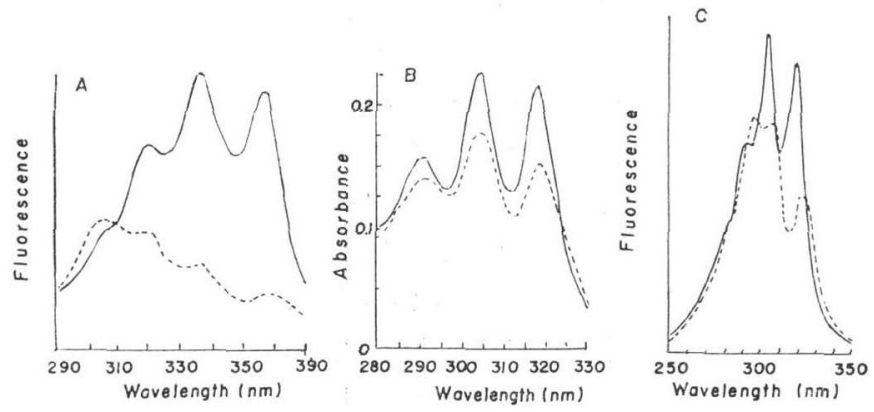
filipin or pimaricin to stigmasterol or cholesterol is $1: 1$ - certainly not 2 or 3 or 0.5 .

Spectral Evidence for Binding

As stated above, another possibility would be that with the conditions used, the fluorescence changes were due to other factors other than binding to sterols. We therefore attempted to determine if the changes in fluorescence (or absorbance for pimaricin) were those that would be expected for interactions with the sterols. These data are shown in Fig. 4. As shown in Fig. 4A, the filipin was binding to cholesterol (the dashed lines) in the presence of pimaricin. The change in spectra is the change expected for binding to sterols ${ }^{6 \sim 8)}$. In this experiment, the solutions contained $3.8 \mu \mathrm{M}$ pimaricin, $3.8 \mu \mathrm{m}$ filipin, and $2.2 \mu \mathrm{m}$ cholesterol. The solid line is the spectra for filipin in the absence of cholesterol but in the presence of pimaricin. This is a normal spectrum for filipin.

The results in Figs. 2C and 2D indicated that amphotericin B was competing very effectively with pimaricin for cholesterol. Therefore, the spectral properties of pimaricin with these experimental conditions were determined. The absorbance spectra of pimaricin in the presence of amphotericin $\mathrm{B}$ (solid line) and in the presence of amphotericin B and cholesterol (dashed line) are given in Fig. 4B. These spectra are similar, if not identical, to spectra that would be obtained if amphotericin $\mathrm{B}$ were not present. We previously reported that pimaricin exhibits increases in fluorescence due to binding to micelles such as lauryl sulfate; however, this was attributed to nonspecific binding ${ }^{15}$. This nonspecific binding can be distinguished by changes in the excitation spectra of pimaricin. Therefore, these experiments were repeated. Pimaricin was mixed with deoxycholate or with amphotericin B and cholesterol, and the excitation spectra was determined. Such an experiment is given in Fig. 4C. The solid line indicates the excitation spectra of pimaricin in the presence of deoxycholate, while the dashed line gives the excitation spectra of pimaricin in the presence of amphotericin B and cholesterol. The interactions are different.

\section{Light Scattering of the Complexes}

Figs. 1 and 2 indicated that both filipin and pimaricin are interacting with sterols to a greater extent than is possible for a 1:1 binding in which the binding to sterol by one polyene in a $1: 1$ complex excludes the binding of the second polyene. If aggregates are formed in which the ratio of polyene antibiotics to sterol is variable, they might be large and detectable by light scattering. We therefore investigated the light scattering properties of different polyene antibiotic sterol mixtures using conditions similar to those used for Figs. 1 and 2. The data (not shown) indicate that the order of addition of components had some effect, but these effects were not large. The data show that large aggregates were not formed. For example, the filipin-cholesterol complex was the same size as the filipin complex, and the filipin plus pimaricin plus cholesterol complex is only slightly larger than the filipin cholesterol complex. With these experimental conditions, the filipin had interacted with the cholesterol as indicated by a $53 \%$ decrease in $\mathrm{CO}$, and the pimaricin had interacted with the cholesterol by the $80 \%$ of maximum change in $\mathrm{CO}$; yet the light-scattering properties of the ternary mixture were very similar to that of controls. Similar results were obtained when the cholesterol was replaced by stigmasterol and the experiments repeated (data also not shown). These light-scattering measurements were performed as described previously ${ }^{14)}$.

\section{Discussion}

The results indicate that the relative affinities of various polyene antibiotics for cholesterol are: 
filipin > amphotericin B > pimaricin > nystatin; and the relative affinities for stigmasterol are: filipin > pimaricin $>$ amphotericin $\mathbf{B}>$ nystatin. The relative affinities for cholesterol are in agreement with the effectiveness of interaction of polyenes for erythrocytes ${ }^{3,6}$.

In these competition experiments, it is not apparent why the amphotericin B did not continue to compete with pimaricin for cholesterol or stigmasterol after the concentration of amphotericin B exceeds $1 / \mathrm{M}$ (see Figs. 2C and 2D). It could be due to the low solubility of amphotericin B in distilled water.

The data show that the stoichiometry of filipin for stigmasterol and cholesterol, and the stoichiometry of pimaricin for stigmasterol or cholesterol is $1: 1$ in dilute $(\mu \mathrm{M})$ aqueous solution. However, the results also indicate that the stoichiometry of amphotericin B for cholesterol or for stigmasterol may be less than $1: 1$. This conclusion is derived from the fact that in Figs. $2 \mathrm{C}$ and $2 \mathrm{D}$, the initial addition of amphotericin $\mathbf{B}$ reduced the fluorescence of the pimaricin-sterol complex to an extent greater than that expected if one sterol molecule was removed per molecule of amphotericin $\mathrm{B}$ added to the solution; i.e., addition of 1.1 nmole of amphotericin $\mathrm{B}$ appeared to remove approximately 2.5 nmoles cholesterol (Fig. 2C) or 2 nmoles stigmasterol from the 11.4 nmoles pimaricin. Thus the ratio of cholesterol or stigmasterol to amphotericin B appear to be greater than $1: 1$. Such ratios have been suggested by others ${ }^{1)}$.

The experiments herein were designed to ensure that the concentration of the polyene antibiotic would be greater than the concentration of sterol; so when a second polyene antibiotic was added, excess sterol would not be present. With these conditions competition between the polyene antibiotics for the sterols should be maximum, and hence changes in fluorescence properties might be proportional to changes in relative amount of sterols bound. The spectral properties of the polyene antibiotics (Figs. $4 \mathrm{~A}, \mathrm{~B}$, and $\mathrm{C}$ ) showed that filipin in the presence of the other polyene antibiotics, and pimaricin in the presence of amphotericin B, formed the expected complexes with cholesterol and stigmasterol. However, the fluorescence data indicate that in the presence of limiting amounts of sterols, both pimaricin and filipin bind to cholesterol to an extent that if the interaction is exclusive and is $1: 1$, more sterol would have to be present in the solutions than had been actually added. The cholesterol concentrations were checked rigorously to ensure that they were indeed correct. This was done by determining the cholesterol concentration with a cholesterol oxidase method (details of the method submitted for publication) and by checking our cholesterol concentrations against those of an independent investigator. The polyene antibiotic concentrations were also rigorously checked using published absorbance spectra. Thus the data indicate that both pimaricin and filipin can interact with cholesterol and stigmasterol when the concentration of cholesterol is limiting. The interaction seems to be such that both antibiotics must be binding to the same sterol molecule. If both polyene antibiotics required a 3- $\beta$ hydroxyl group and the 17-alkyl side chain, then binding to the same sterol molecule would seem unlikely. Yet formation of a ternary complex seems to be a logical explanation. The data in Figs. $3 \mathrm{C}$ and $3 \mathrm{D}$ indicate that after pimaricin interacts with cholesterol or stigmasterol in a $1: 1$ ratio, it continues to bind additional sterols (note the continued increase in $\mathrm{CO}$ ).

Although the light scattering data indicate large aggregates or large ordered structures were not formed in the dilute solutions used in these experiments, formation of moderately large complexes with variable stoichiometries could not be eliminated. However, nonspecific binding of pimaricin to organized structures seems unlikely since the spectral properties are those of pimaricin and/or filipin bound to sterols and not those of the polyene bound to a micelle such as previously shown for sodium deoxycholate $^{15}$.

Acknowledgments

Supported in part by grant No. AI 12187 from the National Institutes of Arthritis and Metabolic Diseases. Paper No. 8569 from the Michigan Agricultural Experiment Station.

\section{References}

1) Norman, A. W.; A. M. Spielvogal \& R. G. Wong: Polyene antibiotic-sterol interaction. Adv. Lipid Res. 14: $127 \sim 170,1976$ 
2) Van Zutphen, H.; R. A. Demel, A. W. Norman \& L. L. M. Van Deenen: The action of polyene antibiotics on lipid bilayer membranes in the presence of several cations and anions. Biochim. Biophys. Acta 241: $310 \sim 330,1971$

3) KInSKy, S. C.: Effect of polyene antibiotics on protoplasts of Neurospora crassa. J. Bacteriol. 83: 351 358,1962

4) KINSKY, S. C.: Comparative response of mammalian erythrocytes and microbial protoplasts to polyene antibiotics and vitamin A. Arch. Biochem. Biophys. 102: 180 188, 1963

5) Cirillo, V. P.; M. Harsch \& J. O. Lampen: Action of polyene antibiotics, filipin, nystatin, and Nacetylcandidin on yeast cell membrane. J. Gen. Microbiol. 35: 249 259, 1964

6) Norman, A. W.; R. A. Demel, B. DeKruyff, W. S. M. Guerts van Kessel \& L. L. M. van Deenen: Studies on the biological properties of polyene antibiotics: Comparison of other polyenes with filipin in their ability to interact specifically with sterol. Biochim. Biophys. Acta 290: 1 14, 1972

7) Norman, A. W.; R. A. Demel, B. DeKruyff \& L. L. M. van Deenen: Studies on the biological properties of polyene antibiotics. J. Biol. Chem. 247: 1918 1929, 1972

8) Bittman, R. \& S. Fischkoff: Fluorescence studies of the binding of the polyene antibiotics filipin III, amphotericin B, nystatin, and lagosin to cholesterol. Proc. Nat. Acad. Sci. USA 69: 3795 3800, 1972

9) Schroeder, F.; J. F. Holland \& L. L. Bieber: Fluorimetric investigation of the interaction of polyene antibiotics with sterols. Biochemistry 11: 3105 3111, 1972

10) Kleinschmidt, M. G.; K. S. Chough \& J. B. Mudd: Effect of filipin on liposomes prepared with different types of sterols. Plant Physiol. 49: 852 859, 1972

11) Bittman, R.; W. C. Chen \& O. R. Anderson: Interaction of filipin III and amphotericin B with lecithin-sterol vesicles and cellular membranes. Spectral and electron microscope studies. Biochemistry 13: $1364 \sim 1373,1974$

12) Bittman, R.; W. C. Chen \& L. Blau: Stopped flow kinetics and equilibrium studies of filipin III binding to sterols. Biochemistry 13: 1374 1379, 1974

13) Lampen, J. O.; P. M. Arnow \& R. S. Safferman: Mechanism of protection by sterols against polyene antibiotics. J. Bact. 80: 200 206, 1960

14) Schroeder, F.; J. F. Holland \& L. L. Bieber: Reversible interconversions of sterol binding and sterol non-binding forms of filipin as determined by fluorimetric and light scattering properties. Biochemistry 12: $4785 \sim 4789,1973$

15) Patterson, Jr., J. M.; R. O. Olinger, J. F. Holland \& L. L. Bieber: Effects of experimental conditions on the interaction of filipin and pimaricin with cholesterol. J. Antibiotics, in press

16) Holland, J. F.; R. E. Teets \& A. Timnick: A unique computer centered instrument for simultaneous absorbance and fluorescence measurements. Anal. Chem. 45: 145 153, 1973 